\title{
Extracorporeal membrane oxygenation support before lung transplant: A bridge over troubled water
}

Gabriel Loor, MD, FACS, ${ }^{\mathrm{a}, \mathrm{b}}$ Subhasis Chatterjee, MD, ${ }^{\mathrm{a}, \mathrm{b}}$ and Alexis Shafii, MD ${ }^{\mathrm{a}, \mathrm{b}}$

Video clip is available online.

Every year, lung transplants save the lives of thousands of patients with irreversible end-stage lung disease. Despite an increased awareness of this condition and a trend toward early referral for transplant, an increasing proportion of patients who present for a lung transplant are critically ill. ${ }^{1,2}$ Even the sickest patients and those who are near death can now benefit from innovative circulatory support strategies that will enable them to survive in good condition and with stable oxygen levels while they wait for an organ to become available. One such strategy, extracorporeal membrane oxygenation (ECMO), allows direct infusion of oxygenated blood into the circulation and clearance of carbon dioxide, irrespective of the condition of the patient's lungs.

In the strictest definition, a bridge-to-transplant (BTT) candidate is a patient receiving ECMO who is deemed by the transplant program's multidisciplinary review board to be a candidate for transplant. Bridging to lung transplant with ECMO is becoming more common as devices become safer, transplant candidates become sicker, and surgeons become more comfortable operating on patients bridged with ECMO. ${ }^{3}$ In addition, since 2005, patients receiving ECMO have been prioritized for lung transplant on the basis of a lung allocation score, which has reduced their time waiting for a lung allograft. Not offering ECMO to a patient with an urgent exacerbation of their lung disease almost always leads to death.

With these factors in mind, we present recent studies that have compared survival outcomes from lung allograft

\footnotetext{
From the a Division of Cardiothoracic Surgery, Michael E. DeBakey Department of Surgery, Baylor College of Medicine, Houston, Tex; and ${ }^{\mathrm{b}}$ Department of Cardiovascular Surgery, Texas Heart Institute, Houston, Tex.

Received for publication Aug 12, 2021; accepted for publication Oct 14, 2021; available ahead of print Nov 9, 2021

Address for reprints: Gabriel Loor, MD, FACS, Department of Cardiovascular Surgery, Texas Heart Institute, 6770 Bertner Ave Suite C-355K, Houston, TX 77030 (E-mail: gabriel.loor@bcm.edu).

JTCVS Open 2021;8:147-54

2666-2736

Copyright (C) 2021 The Author(s). Published by Elsevier Inc. on behalf of The American Association for Thoracic Surgery. This is an open access article under the CC BY-NC-ND license (http://creativecommons.org/licenses/by-nc-nd/4.0/).

https://doi.org/10.1016/j.xjon.2021.10.011
}

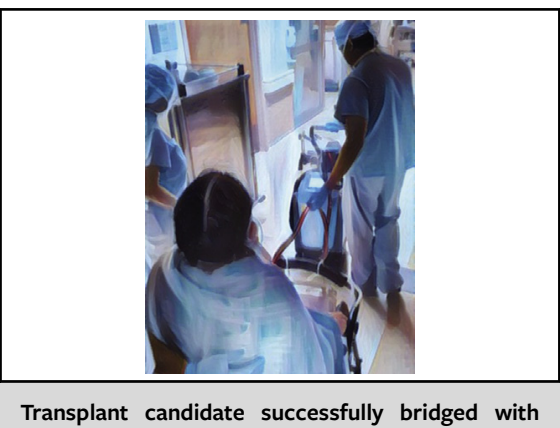

Abiomed Breethe unit for ambulatory ECMO.

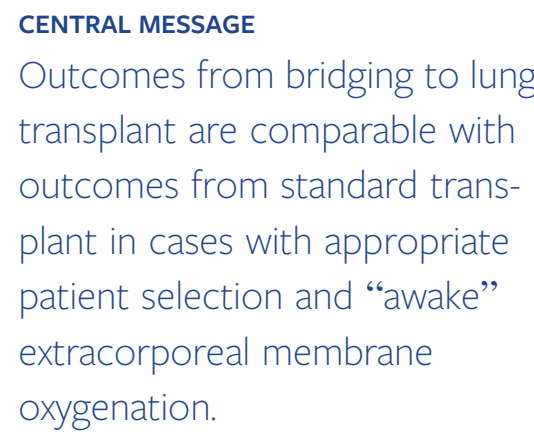

See Commentary on page 155

recipients bridged with ECMO with outcomes from those not bridged with ECMO. We highlight the opportunities that bridging to lung transplant provides and basic principles for achieving a successful outcome. We end with consideration of ECMO in the context of COVID-19. See Video 1 for an overview of these considerations.

\section{ECMO AND LUNG TRANSPLANT IN THE LITERATURE}

Multiple published studies have reported on ECMO as a bridge to lung transplant. Herein, we focus on the 3 most recent single-center experiences (Table 1), because these illustrate the evolution of BTT and associated outcomes in experienced centers. These studies have influenced how we consider BTT for patients with COVID-19, as discussed later in this review.

In a single-center, retrospective analysis of lung transplant patients in Hamburg, Germany, Langer and colleagues ${ }^{4}$ compared the outcomes of 34 ECMO-bridged patients with outcomes of 54 non-bridged patients. Transplants took place from January 2012 to July 2017. The median BTT time was 29 days (range, 0-129 days). Veno- 


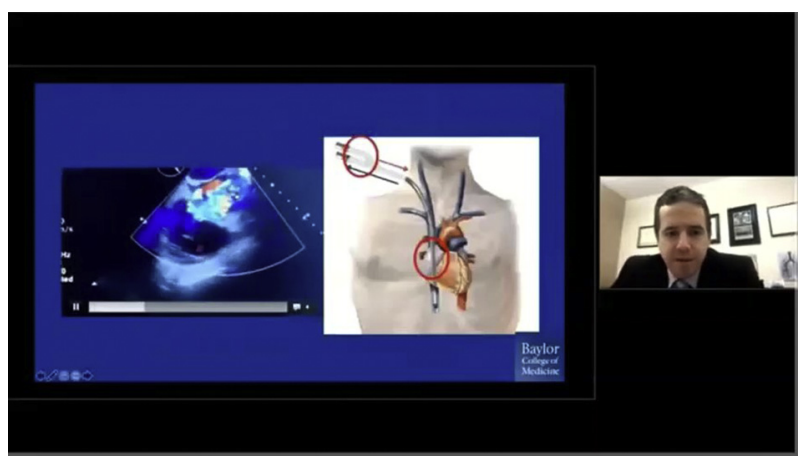

VIDEO 1. In this video, Dr. Gabriel Loor describes innovations, indications, and outcomes for extracorporeal membrane oxygenation as a bridge to lung transplantation. Dr Loor is Surgical Director of Lung Transplant at Baylor St Luke's Medical Center in Houston, Texas. Video available at: https://www.jtcvs.org/article/S2666-2736(21)00362-4/fulltext.

venous (VV) ECMO was used in $96 \%$ of cases, and venoarterial (VA)-venous ECMO was used in $4 \%$ of cases; $18 \%$ of the BTT patients did not require mechanical ventilation during ECMO (ie, "awake ECMO"). The percentage of those who were ambulatory during ECMO (ie, "ambulatory ECMO") was not reported. Post-transplant survival rates were similar among the BTT patients and non-BTT patients (at 1 year, $79 \%$ vs $86 \%$, respectively; at 3 years, $63 \%$ vs $71 \%){ }^{4}$ These results were encouraging, considering the acuity of this patient population.

In another single-center analysis, Tipograf and colleagues $^{5}$ at Columbia University Medical Center reviewed outcomes from patients who underwent lung transplant between 2009 and 2018. Only selected patients who were already on the transplant wait list were deemed candidates for BTT, with few exceptions. Patients with irreversible disease and end-organ damage were often delisted after ECMO was initiated, because of their low chance of survival after transplant. Ultimately, 70/121 ECMO patients (59\%) were successfully bridged to lung transplant. The median BTT time was 12 days; $50 \%$ of patients had VV ECMO. Although most patients required some form of mechanical ventilation, $14 \%$ were never intubated, $20 \%$ had a tracheostomy, and $76 \%$ achieved ambulatory ECMO status. All patients in this series participated in physical therapy, and $71 \%$ had at least 1 day free of mechanical ventilation. The 1-year and 3-year survival rates of $88 \%$ and $83 \%$ for the BTT patients did not differ statistically from those of the 545 non-BTT patients in log rank and propensitymatched analyses. Renal replacement therapy, cerebrovascular accident, and elevated Simplified Acute Physiology II score were risk factors for mortality on the wait list, whereas ambulation was the only factor independently associated with survival to transplant.

Another recent study of BTT patients came from Benazzo and colleagues, ${ }^{2}$ who reviewed 120 patients who met strict criteria for BTT at the Medical University of Vienna over a 20-year period ending in 2017. The study time frame was divided into 3 segments, each encompassing approximately 5 to 7 years. The number of patients who required BTT in the most recent time segment was sevenfold higher than that in the earliest segment; the overall transplant rate among BTT candidates was $89 \%$. Thirteen percent of patients were weaned from mechanical ventilation during ECMO. The study did not report length of time of mechanical ventilation before being weaned, nor did it delineate successful ambulatory ECMO cases.

Survival to discharge after BTT improved steadily over time. The authors noted greater use of VV ECMO over the study period and greater achievement of awake ECMO status. VV ECMO was used in $37 \%$ of all cases, with the rest using VA ECMO, interventional lung assist, VA-venous ECMO, or a combination of strategies. The 5year survival after lung transplant was approximately $63 \%$ for BTT patients and approximately $75 \%$ for nonBTT patients, on the basis of Kaplan-Meier analysis of propensity-matched cohorts. This difference was reported to be statistically significant, although excluding deaths before 90 days eliminated any differences in posttransplant outcomes between the 2 groups. This underscores the importance of patient selection, because BTT patients who have a high likelihood of surviving the first 90 days may expect long-term survival outcomes that are similar to those of non-BTT patients.

\section{ECMO AND POST-TRANSPLANT OUTCOMES}

Patients who undergo lung transplant after ECMO are at high risk for post-transplant complications and require heightened levels of vigilance, resources, and expertise. ${ }^{6}$ Despite the excellent outcomes achieved at experienced

TABLE 1. ECMO as bridge to lung transplant in 3 recent single-center series

\begin{tabular}{lccccccc}
\hline Publication & N* & Median duration, days & Awake, $\%$ & Ambulatory, \% & MV, \% & VV, \% & $\begin{array}{c}\text { Survival 1 y after lung } \\
\text { transplant, \% }\end{array}$ \\
\hline Langer et al. $^{4}$ & 34 & 29 (range, 0-129) & 18 & NA & 82 & 94 & 79 \\
Tipograf et al. $^{5}$ & 70 & $12(\mathrm{IQR}, 5-21)$ & 100 & 76 & $86 \dagger$ & 50 & 88 \\
Benazzo et al. $^{2}$ & 107 & 6 (IQR, 3-14) & 28 & NA & $100 \ddagger$ & 34 & $69 \S$ \\
\hline
\end{tabular}

$M V$, Mechanical ventilation; $V V$, veno-venous extracorporeal membrane oxygenation; $I Q R$, interquartile range; $N A$, not available. *Patients who underwent lung transplant after

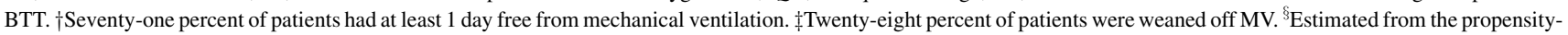
matched cohort comparing BTT with non-BTT. 
lung transplant centers, not all BTT patients can expect similar results. Thankfully, a reasonable amount of published data on prognostic indicators is available to guide physicians in selecting patients for BTT. Many centers that achieve good results with transplants after ECMO will use specific criteria to ensure that the patient has a reasonable chance of survival.

A 2017 publication by our group reported on prognostic factors associated with outcomes after lung transplant in BTT patients. ${ }^{7}$ On the basis of available data and our own experience, we identified the following favorable factors for post-transplant survival after BTT: age younger than 50 years, normal or marginally elevated bilirubin level, normal or mildly elevated pulmonary artery pressures, $<14$-day duration of ECMO, a Sequential Organ Failure Assessment score $<6$, noninvasive ventilation, and the ability to participate in ambulatory ECMO. Unfavorable factors included age older than 60 years, total bilirubin $>3 \mathrm{mg} / \mathrm{dL}$, severe pulmonary hypertension, ECMO duration $>14$ days, prolonged mechanical ventilation, prolonged immobility during ECMO, Sequential Organ Failure Assessment score $>9$, major bleeding, infectious complications, end-organ complications during ECMO, and re-transplant within 1 year.

The observation regarding duration of ECMO support deserves additional mention. This was based on a 2013 case series by Crotti and colleagues, ${ }^{8}$ who showed superior outcomes for patients who underwent transplant with $<14$ days of ECMO support, along with a mortality hazard ratio of 1.12 for each day of ECMO support. Oh and colleagues ${ }^{9}$ used a 14-day cutoff and achieved similar results. Conceivably, prolonged exposure to mechanical circulatory support increases inflammation and coagulopathy, both of which could be detrimental to post-transplant outcomes. However, in our experience and that of others, successful transplant is possible after months of ECMO support (particularly VV ECMO), if the patient is awake, has limited or no mechanical ventilation, is ambulatory, and has stable end-organ perfusion and hemostasis. In fact, during the current COVID-19 pandemic, it has not been uncommon to evaluate patients for transplant after a month of VV ECMO support. The study by Tipograf and colleagues ${ }^{5}$ did not show duration of ECMO support as a prognostic factor for successful BTT; however, their median ECMO duration was only 12 days. Waiting for the best possible donor might offset some of the risk associated with duration of ECMO support. Importantly, considering the scant literature on long-duration ECMO before lung transplant, such cases should be individualized and tailored to the recipient's condition and the center's experience.

Importantly, patients who are awake during ECMO do better, leading to improved BTT results; thus, awake ECMO is the goal for most BTT cases. Although there is no uniformly accepted definition for "awake ECMO," 3 considerations apply: the degree of sedation, the degree of participation with physical therapy, and the need for mechanical ventilation. A fundamental requirement is that the patient should be, at most, only lightly sedated. The awake ECMO patient should be participating in physical therapy, with or without ambulation. If the patient achieves ambulation, the term "ambulatory ECMO" is applied; this is arguably the most encouraging form of awake ECMO. In addition, ECMO without mechanical ventilatory support is often designated as awake ECMO. Although lack of mechanical ventilatory support is a reasonable surrogate for awake ECMO, it is not a requirement for achieving this designation.

Benazzo and colleagues ${ }^{2}$ showed that the ability to achieve awake ECMO was the most important factor associated with post-BTT survival. In this state, patients participated with physiotherapy in bed, were able to move from bed to chair 1 to 2 times per day, and in some cases, were ambulatory. These patients were not always extubated. Langer and colleagues ${ }^{4}$ reported that $100 \%$ of BTT patients who were awake without mechanical ventilation at the time of lung transplant were alive 1 year later. Schechter and colleagues ${ }^{10}$ reported similar post-transplant survival for patients bridged to lung transplant during ECMO without mechanical ventilation and patients who were not bridged with ECMO. Conversely, bridging with ECMO and mechanical ventilation was associated with worse survival. In the study by Tipograf and colleagues, ${ }^{5}$ patients who were ambulatory during ECMO had an odds ratio of 7.5 in favor of surviving to transplant $(95 \% \mathrm{CI}$, 2.15-26.6; $P=.002$ ). Patients who survived to transplant and patients who were not bridged had similar outcomes.

When feasible, we favor extubation without tracheostomy to avoid bleeding and the need for mechanical ventilation. A study by Harris and colleagues ${ }^{11}$ in a nontransplant population showed a small but significant increase in blood transfusion rates in patients receiving VV ECMO with tracheostomy versus those without tracheostomy. Tracheostomy before lung transplant is sometimes unavoidable because of respiratory secretion accumulations and muscle weakness. When tracheostomy is necessary, we hold anticoagulation for at least 8 hours before the procedure and for 24 hours after the procedure when feasible. The use of anticoagulation in VV ECMO is controversial and is discussed further in the section "ECMO Management Challenges." We increase the ECMO flow rates to at least $3 \mathrm{~L} / \mathrm{min}$ during this time to avoid clotting. In addition, even with tracheostomy, we try aggressively to maintain patients in an awake ECMO state with light sedation and participation in physiotherapy directed by dedicated physical therapy teams. We avoid mechanical ventilation whenever possible and wean the patient to pressure support and a tracheal collar as soon as possible. 
Habertheuer and colleagues ${ }^{12}$ at The University of Pennsylvania developed and validated a useful score for identifying post-transplant mortality risk for BTT patients. Using data from 822 BTT lung transplant patients in the United Network for Organ Sharing database, the group used a linear prediction method to construct the Stratification Risk Analysis in Bridging Patients to Lung Transplant on ECMO (STABLE) score. The area under the curve for this tool was $89 \%$, suggesting that it is an accurate prediction model. Univariate and multivariate analyses identified age older than 50 years, $>75$ days on the wait list, dialysis while on the wait list, mechanical ventilation while on the wait list, and total bilirubin $>1.2 \mathrm{mg} / \mathrm{dL}$ as predictive of post-transplant mortality. The presence of each of these 5 factors is assigned a weighted score (Table 2). The lowest score is 0 (best outcome) and the highest score is 24 (worst outcome). The higher the score, the greater the odds of mortality; for instance, a score of 0 is associated with a $3 \%$ chance of in-hospital mortality after transplant, whereas a score of 24 indicates a $78 \%$ chance.

Whether bridging a patient with VA versus VV ECMO support is associated with different outcomes after lung transplant is unknown. However, VV ECMO is the method of choice for isolated respiratory failure. ${ }^{13} \mathrm{Few}$ studies have compared VA versus VV bridging outcomes after lung transplant. Generally, BTT patients who require VA ECMO have severe pulmonary hypertension and right ventricular dysfunction. These patients are known to have worse outcomes after lung transplant than patients with

TABLE 2. Factors comprising the STABLE scoring system for predicting mortality risk after lung transplant

\begin{tabular}{|c|c|c|}
\hline Factor & Value & $\begin{array}{c}\text { STABLE } \\
\text { points }\end{array}$ \\
\hline \multirow[t]{2}{*}{ Age } & $18-50$ years & 0 \\
\hline & $>50$ years & 3 \\
\hline \multirow[t]{2}{*}{ Time on wait list } & 1-75 days & 0 \\
\hline & $>75$ days & 5 \\
\hline \multirow[t]{2}{*}{ Dialysis on the wait list } & No & 0 \\
\hline & Yes & 6 \\
\hline \multirow[t]{2}{*}{ Transplant center volume } & $\begin{array}{l}\geq 50 \text { lung transplants } \\
\text { per year }\end{array}$ & 0 \\
\hline & $\begin{array}{c}<50 \text { lung transplants } \\
\text { per year }\end{array}$ & 3 \\
\hline \multirow{2}{*}{$\begin{array}{l}\text { Mechanical ventilation on } \\
\text { wait list }\end{array}$} & No & 0 \\
\hline & Yes & 4 \\
\hline \multirow[t]{2}{*}{ Total bilirubin } & $\leq 1.2 \mathrm{mg} / \mathrm{dL}$ & 0 \\
\hline & $>1.2 \mathrm{mg} / \mathrm{dL}$ & 3 \\
\hline Total points & & 24 \\
\hline
\end{tabular}

other indications for listing. VA ECMO is certainly warranted in patients who are decompensating with $\mathrm{VV}$ ECMO support because of worsening hemodynamics. ${ }^{2}$ Thus, VA ECMO probably indicates more severe disease progression that might be associated with worse posttransplant outcomes. Tipograf and colleagues ${ }^{5}$ reported that $50 \%$ of patients were bridged with VV ECMO and $46 \%$ were bridged with VA ECMO, but no comparison of outcomes was provided. Outcomes after lung transplant are most likely related to the same factors in both modalities (ie, ambulatory status, degree of critical illness, and other patient comorbidities).

There are several reasons for the increased adoption of VV ECMO over VA ECMO for bridging to lung transplant. The modern VV cannulas for ambulatory ECMO are duallumen with a single insertion site. This considerably facilitates ambulation, although maintaining adequate flows and oxygenation can be difficult at times. VA ECMO exposes the patient to risks from the outflow cannula in the arterial circulation, including embolic events, stroke, and limb complications. Because the outflow in VA ECMO is directly in line with the arterial circulation, greater amounts of anticoagulation are often needed. Bridging with VA ECMO is very acceptable so long as an ambulatory arrangement can be selected. The VA circuit should be configured to ensure a good likelihood of wakefulness while maintaining good oxygenation and organ perfusion. Vascular or bleeding complications should be anticipated and avoided if at all possible.

Finally, center volume is an important determinant of outcomes. In an analysis of United Network for Organ Sharing data, Hayanga and colleagues ${ }^{14}$ reported that low-volume centers $(<5$ transplants per year) had a $2.74-$ times higher risk-adjusted 1-year mortality rate, compared with high-volume centers ( $>15$ transplants per year). Similar findings were noted in the development of the STABLE score. ${ }^{12}$

\section{ECMO TECHNICAL CONSIDERATIONS}

The ultimate goal during ECMO is for patients to be ambulatory and awake. It is not always possible to achieve this initially, for a variety of reasons. If the patient is critically ill and needs ECMO urgently, standard VV insertion with oxygenated outflow in the upper-extremity veins and deoxygenated inflow from the femoral veins is usually the safest and fastest method. ${ }^{7}$ Cannulation can be done at the bedside, with or without fluoroscopy. If fluoroscopy is not available, transthoracic or transesophageal echocardiography should be used to guide wire and cannula placement.

After the patient is stabilized, achieving awake ECMO is easier if the standard VV cannulas are replaced with a single dual-lumen cannula in the neck. Moreover, if a patient is considered for BTT early and is not initially unstable, it is reasonable to use a single dual-lumen cannula from the 
beginning. Dual-lumen cannulas should be inserted in a hybrid room and with fluoroscopic and transesophageal echocardiographic guidance.

Either the internal jugular vein (IJ) or the subclavian vein can be cannulated. We first described placement of the Avalon cannula via the left subclavian vein and continue to find this approach advantageous, for several reasons. ${ }^{15}$ First, the subclavian approach enhances cannula stability and is more comfortable for the patient while awake and ambulating. Second, a subclavian cannula remains less mobile during ambulation. In the IJ configuration, movement of the cannula can interfere with establishing stable partial pressure of oxygen. Ideally, the outflow is directed as close as possible to the tricuspid valve, but even a few millimeters of movement can direct the oxygenated outflow from a dual-lumen cannula away from the tricuspid valve, leading to recirculation. Transesophageal echocardiographic guidance, having a pulmonary artery catheter in place, and radiopaque markers on the cannula can help ensure correct orientation.

It is worthwhile to obtain contrast-enhanced computed tomographic or ultrasound imaging to ensure that the subclavian vein is large enough for the cannula. Contrast through the brachial vein can help. The subclavian approach can be hazardous, because the cannula has to traverse the innominate vein and angle down toward the inferior vena cava. Nonetheless, we have not encountered any complications when using this tactic, and it is our preferred method in patients with suitable anatomy.

When we do need to cannulate the IJ, we sometimes wrap a ringed 10-mm GORE-TEX (W. L. Gore \& Associates, Inc) vascular graft around the cannula and sew it to the dermal/subcutaneous edge of the skin incision after the cannula has been positioned correctly under fluoroscopic guidance. We sew this with a running 4-0 Prolene suture and place several ties around it, followed by anchoring sutures to the skin. This stabilizes the cannula.

After an upper-extremity VV circuit has been established, we focus on reducing sedation and waking the patient. We extubate patients if possible, but if they are too weak to control their secretions, a tracheostomy is reasonable. We will usually stagger these 2 procedures to reduce bleeding complications. We have been able to support patients for up to 9 months before transplantwith this configuration-albeit not without hazards, including air entry into the cannula (which requires urgent exchange), bleeding (which can be addressed by holding anticoagulation and placing a suture at the bedside), and bacteremia.

One of the most feared complications is stroke. Stroke was reported to occur in $5.9 \%$ of VV ECMO patients and $8.8 \%$ of VA ECMO patients with acute respiratory distress syndrome (ARDS). ${ }^{13}$ Stroke occurred in $8.3 \%$ of all attempted BTT cases in the series by Tipograf and colleagues, ${ }^{5}$ which included VA and VV ECMO cases in approximately equal proportions.

If a patient has worsening right ventricular dysfunction, we prefer an atrial septostomy approach when feasible. ${ }^{16-}$

${ }^{18}$ However, in cases of severe pulmonary hypertension this might not be sufficient; VA ECMO should then be considered. Femoral VA ECMO is the strategy of choice for the acute decompensated patient with pulmonary hypertension, although after the patient is stabilized, it is ideal to convert to a fully ambulatory arrangement in the operating room, if possible.

For VA ECMO, we have adopted a modified version of the "sport model." 19 Our preference is to leave the chest closed by placing a 17-French Biomedicus arterial cannula (Medtronic) through a hand-sewn, 10-mm Gelweave graft on the right subclavian artery. Using fluoroscopy, we position the tip of the cannula into the innominate artery to prevent limb hyperperfusion. We then cannulate the right IJ by using a Seldinger technique with a 29/29/29 triple-stage atrial cannula (Medtronic) for drainage. This requires insertion of a dilator through the cannula to allow it to safely traverse the soft tissue. With this configuration, we have supported BTT patients with severe pulmonary hypertension for up to 20 days with ambulation, no tracheostomy, and no limb complications. Of note, if a patient is tolerating femoral VA ECMO with adequate oxygenation and no limb ischemia, it is not necessary to alter this arrangement for ambulation. $^{20}$

To achieve ambulatory VA ECMO, it might be necessary to directly cannulate the aorta and atrium through a limited right thoracotomy, mini upper sternotomy, or full sternotomy. ${ }^{19,21}$ The downsides of this approach are infection risk, bleeding risk, and formation of adhesions. Invasive cannulation strategies put some patients, such as those with severe pulmonary hypertension or hepatic venous congestion, at particularly high risk for bleeding.

Moreover, the surgeon performing the lung transplant can use the existing cannulas and ECMO arrangement for the procedure, so long as the hemodynamics are supported. In our experience, we are rarely able to maintain a patient by using VV ECMO alone, although we will attempt to do so with a pulmonary artery test clamp. More commonly, we switch to VA ECMO by centrally cannulating the aorta and introducing a second venous multistage cannula through the groin. We find that a dual-lumen single-stage cannula alone is not sufficient for drainage. In addition, we push intravenous fluids through the outflow (oxygenated) port while preventing air intake, to keep the duallumen cannula useable throughout the procedure. We typically remove the femoral venous cannula after the transplant and leave the dual-lumen single cannula in place overnight. If ECMO is not sufficient for support, we use cardiopulmonary bypass. 


\section{ECMO AND COVID-19}

Since early 2020, the COVID-19 pandemic has dramatically transformed all of health care and the world at large. The availability of ECMO has offered the possibility of recovery for thousands of patients worldwide, ${ }^{22}$ including those who need a lung transplant for COVID-19-related respiratory failure and pulmonary fibrosis. ${ }^{23}$ The fundamental principles outlined previously are being used for BTT patients receiving ECMO because of COVID-19 respiratory failure. At our own center, we have performed nearly a dozen lung transplants in COVID-19 patients, with $100 \%$ perioperative survival to discharge, and we anticipate more to come.

Patients who require transplant because of COVID-19 ARDS are often receiving ECMO support. Generally, they are patients who have not regained respiratory function after 4 to 6 weeks despite clearance of the SARS-CoV-2 virus. In a recent review, the median time in hospital for these patients before transplant was 71 days, with a wide range of 30 to 125 days. ${ }^{24}$ It is not uncommon for most of this time to be spent receiving some sort of ECMO support. Generally, we apply the same principles outlined previously to care for patients bridging to lung transplant because of COVID-19, with a few caveats: (1) achieving awake status is necessary before a patient can be listed for transplant at our institution; this can be difficult to achieve and generally takes considerably longer than usual. (2) Patients might be more susceptible to prothrombotic states and require more heightened vigilance with respect to anticoagulation, which might expose them to greater bleeding risks than the average BTT patient. (3) Patients might be more susceptible to multiorgan dysfunction, including liver, renal, cardiac, and neurological toxicities.

Patients with COVID-19 differ considerably from patients with chronic respiratory illness, yet they can be good candidates for BTT so long as they meet the typical indications for transplant, including irreversible lung disease without other end-organ dysfunction, and are cleared for transplant by the medical review board. In a recent series from Bharat and colleagues, ${ }^{25}$ explanted COVID-19 lungs had severe fibrotic changes, indicating that the disease can become irreversible and require a transplant. Because many of these patients have not had preexisting major comorbidities, they might be more tolerant of the stresses of ECMO and might actually do very well after lung transplant.

In another recent multicenter series, Bharat and colleagues ${ }^{23}$ reported their experience with 12 patients bridged to lung transplant for COVID-19-related ARDS. All but 1 patient achieved awake ECMO status. Eleven of the 12 patients were alive at follow-up, including the patient who could not achieve awake ECMO status before transplant. This brings up several ethical and outcomes issues. On the ethics front, if the sedated patient has not already consented to the transplant, it is important to obtain consent from someone who knows the patient best and can attest to the patient's wishes. This will certainly require individual program-specific ethical review. We have not performed a transplant without expressly discussing it with the patient first. On the outcomes front, ambulation or at least an awake ECMO state substantially improves the likelihood of a successful outcome. That does not mean that the outcome without ambulation is prohibitively poor.

No exclusions or modifications specific to COVID-19 cases have been developed with respect to a transplant center's 1-year survival reporting. Each center will need to develop its own risk tolerance to ensure that its program meets acceptable benchmarks for postoperative survival. Partnering with other high-volume centers is a reasonable way to leverage additional resources and expertise.

\section{ECMO MANAGEMENT CHALLENGES}

Anticoagulation for patients receiving ECMO involves balancing the risk for thrombotic complications within the circuit against the risk for bleeding complications. In our practice, unfractionated heparin is the first-line anticoagulation agent; we use a weight-based nomogram and aim for an activated partial thromboplastin time of 40 to 55 seconds, consistent with major randomized VV ECMO trials. ${ }^{26}$ We monitor with activated partial thromboplastin time and the thromboelastogram to aim for concordance; if there is discordance between these 2, we use anti-factor Xa monitoring. ${ }^{27}$ Some centers have moved toward early initiation of direct thrombin inhibitors, which is a reasonable strategy. In our practice, we maintain a low threshold for using direct thrombin inhibitors when we suspect heparin-induced thrombocytopenia. ${ }^{28}$ Moreover, if ECMO flows are adequate, we are conservative with holding anticoagulation for a period of time as clinically indicated.

Patients with ARDS often are deeply sedated and undergo neuromuscular blockade before VV ECMO initiation. Postoperatively, strategies to reduce the risks associated with prolonged mechanical ventilation after mechanical circulatory support include adherence to the ABCDEF bundle. ${ }^{29}$ For patients with ARDS and COVID-19, multiple reports have shown that very high doses of analgesia and sedation are required. The reasons proposed for this phenomenon include younger patient age, high respiratory drive, and an intense inflammatory response that results in medication tolerance. ${ }^{30,31}$ Patients are typically weaned off neuromuscular blockade in the first 24 hours after initiation of VV ECMO. In our experience, higher doses of quetiapine (400-600 mg/d), earlier tracheostomy, and accepting a lower oxygen saturation level $(80 \%)$ with normal lactate level might facilitate weaning from sedation in this challenging patient cohort. Nevertheless, weaning from neuromuscular blockade and 
deep sedation might take more time, occasionally 1 to 2 months. For the reasons discussed previously, it is difficult, although not unheard of, to offer transplant to patients in this condition.

The minimum criteria for a patient to remain on the transplant list when they are bridged to lung transplant are specific to the practice patterns and risk tolerance of individual institutions. As the STABLE score underscores, accumulation of risk factors increases the odds of mortality after lung transplant. Although there is no single threshold to which all centers adhere, when a treatment team believes that the chances of a good outcome are futile or that the outcome is not what the patient would consider a meaningful recovery, the patient should be removed from the wait list. In our center, we see irreversible injuries such as stroke, renal failure, or liver failure as reasons to delist. We would still give the patient time to see if the injuries resolve, but in most cases they do not. Infection and sepsis are other reasons for delisting. Major bleeding or cannulation issues also are important. Escalating from VV to VA ECMO is not necessarily a reason to delist, but it does potentially add risk.

The ideal candidate on the waitlist is awake, responsive, participatory with basic physical therapy (ie, sitting up in a chair, at least), has preserved end-organ function (including kidney, liver, and brain), and lacks evidence of active infection. Although there is no set point to delist, these cases are reviewed weekly in a multidisciplinary format. We do not delist a patient for inactivity, but if over time we see multiple consecutive days of inactivity (ie, $>5$ days) accompanied by malnutrition and worsening frailty, we consider delisting. If persistent inactivity or worsening clinical status is noted, the patient is removed from the list. Routine palliative care consultation is recommended for these patients and families, to promote thoughtful decision-making consistent with the patient's wishes.

\section{CONCLUSIONS}

The use of ECMO as a BTT is a reality of modern-day lung transplant and is likely to become increasingly common, especially because of the COVID-19 pandemic. By using reasonable acceptance criteria, experienced centers can expect similar long-term survival for BTT patients compared with non-BTT patients, albeit with greater resource utilization. Ambulatory or awake ECMO should be the goal of any bridging strategy. Innovation in this area will undoubtedly evolve to include durable compact circuits and novel cannulation options, along with methods to reduce risk for bleeding and neurological complications during ECMO.

\section{Conflict of Interest Statement}

G.L. reports institutional grant support to Baylor College of Medicine from Abiomed, Transmedics, and Maquet for research related to ECMO, ex vivo lung perfusion, and lung transplant. G.L. and A.S. report institutional grant support to Baylor College of Medicine from Abbott for left ventricular assist device research. G.L. is a consultant for Abiomed Breethe and a scientific advisor for Transmedics. S.C. has served on advisory boards for Edwards Lifesciences, La Jolla Pharmaceuticals, and Baxter. Several practices discussed in this narrative include off-label use of US Food and Drug Administration-approved devices, given the novelty and evolving techniques in ECMO and lung transplant.

The Journal policy requires editors and reviewers to disclose conflicts of interest and to decline handling or reviewing manuscripts for which they may have a conflict of interest. The editors and reviewers of this article have no conflicts of interest.

The authors' studies discussed in this narrative were conducted under the Baylor College of Medicine Institutional Review Board protocol H-41620, Baylor St. Luke's Lung Transplant Database for the Study of Lung Transplant Outcomes, approved August 2017 with waiver of consent.

Jeanie F. Woodruff, BS, ELS, of the Scientific Publications \& Grants Department at the Texas Heart Institute, contributed to the editing of the manuscript.

\section{References}

1. Valapour M, Lehr CJ, Skeans MA, Smith JM, Uccellini K, Goff R, et al. OPTN/ SRTR 2018 annual data report: lung. Am J Transplant. 2020;20:427-508.

2. Benazzo A, Schwarz S, Frommlet F, Schweiger T, Jaksch P, Schellongowski P, et al. Twenty-year experience with extracorporeal life support as bridge to lung transplantation. J Thorac Cardiovasc Surg. 2019;157:2515-25.e10.

3. Mattar A, Chatterjee S, Loor G. Bridging to lung transplantation. Crit Care Clin. 2019;35:11-25.

4. Langer F, Aliyev P, Schäfers HJ, Trudzinski FC, Seiler F, Bals R, et al. Improving outcomes in bridge-to-transplant: extended extracorporeal membrane oxygenation support to obtain optimal donor lungs for marginal recipients. ASAIO J. 2019;65:516-21.

5. Tipograf Y, Salna M, Minko E, Grogan EL, Agerstrand C, Sonett J, et al. Outcomes of extracorporeal membrane oxygenation as a bridge to lung transplantation. Ann Thorac Surg. 2019;107:1456-63.

6. Shafii AE, Mason DP, Brown CR, Vakil N, Johnston DR, McCurry KR, et al. Growing experience with extracorporeal membrane oxygenation as a bridge to lung transplantation. ASAIO J. 2012;58:526-9.

7. Loor G, Simpson L, Parulekar A. Bridging to lung transplantation with extracorporeal circulatory support: when or when not? J Thorac Dis. 2017;9: 3352-61.

8. Crotti S, Iotti GA, Lissoni A, Belliato M, Zanierato M, Chierichetti M, et al. Organ allocation waiting time during extracorporeal bridge to lung transplant af fects outcomes. Chest. 2013;144:1018-25.

9. Oh DK, Hong SB, Shim TS, Kim DK, Choi S, Lee GD, et al. Effects of the duration of bridge to lung transplantation with extracorporeal membrane oxygenation. PLoS One. 2021;16:e253520.

10. Schechter MA, Ganapathi AM, Englum BR, Speicher PJ, Daneshmand MA, Davis RD, et al. Spontaneously breathing extracorporeal membrane oxygenation support provides the optimal bridge to lung transplantation. Transplantation. 2016;100:2699-704.

11. Harris DD II, Shafii AE, Baz M, Tribble TA, Ferraris VA. Increased blood transfusion and its impact in patients having tracheostomy while on extracorporeal membrane oxygenation. Perfusion. 2019;34:143-6.

12. Habertheuer A, Richards T, Sertic F, Molina M, Vallabhajosyula P, Suzuki Y, et al. Stratification risk analysis in bridging patients to lung transplant on ECMO: the STABLE risk score. Ann Thorac Surg. 2020;110:1175-84.

13. Kon ZN, Bittle GJ, Pasrija C, Pham SM, Mazzeffi MA, Herr DL, et al. Venovenous versus venoarterial extracorporeal membrane oxygenation for adult 
patients with acute respiratory distress syndrome requiring precannulation hemodynamic support: a review of the ELSO Registry. Ann Thorac Surg. 2017; 104:645-9.

14. Hayanga JW, Lira A, Aboagye JK, Hayanga HK, D’Cunha J. Extracorporeal membrane oxygenation as a bridge to lung transplantation: what lessons might we learn from volume and expertise? Interact Cardiovasc Thorac Surg. 2016; 22:406-10.

15. Shafii AE, McCurry KR. Subclavian insertion of the bicaval dual lumen cannula for venovenous extracorporeal membrane oxygenation. Ann Thorac Surg. 2012; 94:663-5.

16. Camboni D, Akay B, Sassalos P, Toomasian JM, Haft JW, Bartlett RH, et al. Use of venovenous extracorporeal membrane oxygenation and an atrial septostomy for pulmonary and right ventricular failure. Ann Thorac Surg. 2011;91:144-9.

17. Hoopes CW, Gurley JC, Zwischenberger JB, Diaz-Guzman E. Mechanical support for pulmonary veno-occlusive disease: combined atrial septostomy and venovenous extracorporeal membrane oxygenation. Semin Thorac Cardiovasc Surg. 2012;24:232-4.

18. Kon ZN, Pasrija C, Shah A, Griffith BP, Garcia JP. Venovenous extracorporeal membrane oxygenation with atrial septostomy as a bridge to lung transplantation. Ann Thorac Surg. 2016;101:1166-9.

19. Biscotti M, Bacchetta M. The "sport model": extracorporeal membrane oxygenation using the subclavian artery. Ann Thorac Surg. 2014;98:1487-9.

20. Pasrija C, Mackowick KM, Raithel M, Tran D, Boulos FM, Deatrick KB, et al. Ambulation with femoral arterial cannulation can be safely performed on venoarterial extracorporeal membrane oxygenation. Ann Thorac Surg. 2019;107: 1389-94.

21. Downey P, Ragalie W, Gudzenko V, Ardehali A. Ambulatory central venoarterial extracorporeal membrane oxygenation in lung transplant candidates. $J$ Heart Lung Transplant. 2019;38:1317-9.
22. Barbaro RP, MacLaren G, Boonstra PS, Iwashyna TJ, Slutsky AS, Fan E, et al. Extracorporeal membrane oxygenation support in COVID-19: an international cohort study of the Extracorporeal Life Support Organization Registry. Lancet. 2020;396:1071-8.

23. Bharat A, Machuca TN, Querrey M, Kurihara C, Garza-Castillon R Jr, Kim S, et al. Early outcomes after lung transplantation for severe COVID-19: a series of the first consecutive cases from four countries. Lancet Respir Med. 2021;9:487-97.

24. Hawkins RB, Mehaffey JH, Charles EJ, Mannem HC, Roeser M. Lung transplantation for severe post-coronavirus disease 2019 respiratory failure. Transplantation. 2021;105:1381-7.

25. Bharat A, Querrey M, Markov NS, Kim S, Kurihara C, Garza-Castillon R, et al. Lung transplantation for patients with severe COVID-19. Sci Transl Med. 2020; 12:eabe4282.

26. Combes A, Hajage D, Capellier G, Demoule A, Lavoué S, Guervilly C, et al. Extracorporeal membrane oxygenation for severe acute respiratory distress syndrome. N Engl J Med. 2018;378:1965-75.

27. Colman E, Yin EB, Laine G, Chatterjee S, Saatee S, Herlihy JP, et al. Evaluation of a heparin monitoring protocol for extracorporeal membrane oxygenation and review of the literature. J Thorac Dis. 2019;11:3325-35.

28. Patel B, Diaz-Gomez JL, Ghanta RK, Bracey AW, Chatterjee S. Management of extracorporeal membrane oxygenation for postcardiotomy cardiogenic shock. Anesthesiology. 2021;135:497-507.

29. Ely EW. The ABCDEF bundle: science and philosophy of how ICU liberation serves patients and families. Crit Care Med. 2017;45:321-30.

30. Kapp CM, Zaeh S, Niedermeyer S, Punjabi NM, Siddharthan T, Damarla M. The use of analgesia and sedation in mechanically ventilated patients with COVID-19 acute respiratory distress syndrome. Anesth Analg. 2020;131:e198-200.

31. Hanidziar D, Bittner EA. Sedation of mechanically ventilated COVID-19 patients: challenges and special considerations. Anesth Analg. 2020;131:e40-1. 